\title{
Tight Bound on Parameter of Surplus-Based Averaging Algorithm over Balanced Digraphs
}

\author{
Satoshi Kawamura ${ }^{1}$, Kai Cai ${ }^{2}$, Mengbin $\mathrm{Ye}^{3}$, and Zhiyun $\mathrm{Lin}^{4}$
}

\begin{abstract}
We study a continuous-time surplus-based algorithm for multi-agent average consensus, and derive a tight upper bound on the key parameter included in this algorithm that ensures convergence over strongly connected and balanced digraphs. We specialize the upper bound result to undirected (connected) graphs and unweighted cyclic digraphs; in particular, for undirected graphs the algorithm converges for arbitrary positive values of the parameter, and for cyclic digraphs the upper bound on the parameter depends only on the number of agents and may be easily calculated. Moreover, it is suggested through extensive simulation that, for the same number of agents, the upper bound for cyclic digraphs be smaller than that for other strongly connected and possibly unbalanced digraphs; this implies that as long as the parameter satisfies the upper bound for cyclic digraphs, this parameter can work for other digraphs.
\end{abstract}

\section{INTRODUCTION}

The problem of multi-agent average consensus has attracted significant attention in the systems and control community (Jadbabaie, Lin, \& Morse, 2003; Olfati-Saber \& Murray, 2004; Xiao \& Boyd, 2004;

Satoshi Kawamura and Kai Cai are supported in part by the Research and Development of Innovative Network Technologies to Create the Future of National Institute of Information and Communications Technology (NICT) of Japan, and the open collaborative research program at National Institute of Informatics (NII) Japan (FY2018). Mengbin Ye is supported in part by the European Research Council (ERCCoG-771687) and the Netherlands Organization for Scientific Research (NWO-vidi14134).

${ }^{1}$ Satoshi Kawamura is with Dept. of Electrical and Information Engineering, Osaka City University, Japan. kawamura@c.info.eng.osaka-cu.ac.jp

${ }^{2} \mathrm{Kai}$ Cai is with Dept. of Electrical and Information Engineering, Osaka City University, Japan. kai.cai@eng.osaka-cu.ac.jp

${ }^{3}$ Mengbin Ye is with the Faculty of Science and Engineering, ENTEG, University of Groningen, Groningen 9747 AG, Netherlands. m.ye@rug.nl

${ }^{4}$ Zhiyun Lin is with School of Automation, Hangzhou Dianzi University, China. linz@hdu.edu.cn
Boyd, Ghosh, Prabhakar, \& Shah, 2006; Bénézit, Blondel, Thiran, Tsitsiklis, \& Vetterli, 2010). In this problem, a network of interconnected agents aims to reach an agreement on the average value of their initial states, through only local communications among neighbors. The inter-agent communication topology is modeled by a directed graph (or digraph). Average consensus has found applications in multi-vehicle cooperation, distributed estimation, load balancing, and distributed optimization (Cao, Yu, Ren, \& Chen, 2013; Qin, Ma, Shi, \& Wang, 2017).

In (Olfati-Saber \& Murray, 2004) a basic consensus algorithm was introduced but the consensus value depends on the communication topology and the agents' initial states. By contrast, in (Cai \& Ishii, 2012) a "surplus-based" algorithm was proposed which provably achieves average consensus on arbitrary strongly connected (time-invariant) digraphs. In this algorithm, a variable called "surplus" is augmented to each agent, which tracks the state changes of the associated agent; collectively these surplus variables keep the information of state sum shift, thereby achieving average consensus even if the digraph is not balanced (the latter requires that each agent maintains the same amount of incoming and outgoing information). The surplus-based algorithm has been extended to address average consensus on random networks (Cai, 2012), time-varying networks (Cai \& Ishii, 2014), and to solve a distributed optimization problem with state constraints (Xu et al., 2017) (these works have mainly been on discrete-time algorithms).

Despite many developments of the surplus-based algorithm, a fundamental issue remains unsolved. There is a parameter in the algorithm, whose amount must be "sufficiently small" in order to ensure the convergence of the algorithm. An explicit and tight bound on the parameter is yet unknown. For the discrete-time surplus-based algorithm, Cai and Ishii (2012) presented an explicit, but highly conservative upper bound based on a matrix perturbation result. Even for several special digraphs (balanced, cyclic, 
and undirected), the bounds reported in (Cai \& Ishii, 2012) are not tight. For the continuous-time version in (Cai, 2011), there has not been any bound result reported.

In this paper, we study upper bounds for the parameter in the continuous-time surplus-based algorithm. In order to derive tight bounds, we focus on balanced digraphs and directly analyze the eigenvalues of the multi-agent system as functions of the parameter. Our contributions are threefold:

1. We derive a tight upper bound for the parameter for strongly connected and balanced digraphs, which is explicit in terms of the real and imaginary parts of the graph Laplacian's eigenvalues.

2. For undirected (connected) graphs we show that the continuous-time surplus-based algorithm converges for any positive value of the parameter. This is in contrast with the parameter bound for the discrete-time algorithm (Cai \& Ishii, 2012), which is dependent on the number of agents in the network.

3. For cyclic digraphs we derive an explicit and tight bound that depends only on the number of agents and is easily calculatable. This is an improved result as compared to the conservative bound given in (Cai \& Ishii, 2012) for the discrete-time algorithm.

In addition, we show by simulation that, for the same number of agents, the bound for cyclic digraphs is plausibly smaller than that for any other strongly connected and unbalanced digraphs; this means that as long as the parameter satisfies the (tight) bound for cyclic digraphs (which is explicitly known), the surplus-based algorithm with this parameter value may work for other possibly unbalanced digraphs.

The rest of this paper is organized as follows. Section 2 introduces communication graphs and the continuous-time surplus-based average consensus algorithm. Sections 3 and 4 present the main results; tight upper bounds on the parameter are derived for balanced, undirected, and cyclic digraphs. Section 5 provides illustrative simulation examples, and finally Section 6 states our conclusions.

\section{PRELIMINARIES}

In this paper, we will use the following notation. Denote $\mathbb{R}_{+}$as the set of positive real numbers, $I_{n}$ the $n \times n$ identity matrix, $O_{n}$ the $n \times n$ zero matrix, $\mathrm{j}$ (roman) the imaginary unit, i.e. $\mathrm{j}:=\sqrt{-1}$. Also define $\mathbf{0}_{n}:=[0 \cdots 0]^{\top} \in \mathbb{R}^{n}$ and $\mathbf{1}_{n}:=[1 \cdots 1]^{\top} \in \mathbb{R}^{n}$.

\subsection{Communication graphs}

Given a multi-agent system, we represent the multiagent system and the interactions between the agents by a communication graph $\mathcal{G}=(\mathcal{V}, \mathcal{E})$, with node set $\mathcal{V}=\left\{v_{1}, \ldots, v_{n}\right\}$ and directed edge set $\mathcal{E} \subseteq \mathcal{V} \times \mathcal{V}$. The graph is assumed to be directed, unweighted, and time-invariant. The node $v_{i} \in \mathcal{V}$ represents the $i$ th agent. The $i$ th agent receives information from the $j$ th agent if and only if $\left(v_{j}, v_{i}\right) \in \mathcal{E}$. We define the set of "in-neighbors" of the $i$ th agent by $\mathcal{N}_{i}^{\mathrm{I}}:=$ $\left\{v_{k} \mid\left(v_{k}, v_{i}\right) \in \mathcal{E}\right\}$ and the set of "out-neighbors" by $\mathcal{N}_{i}^{\mathrm{O}}:=\left\{v_{k} \mid\left(v_{i}, v_{k}\right) \in \mathcal{E}\right\}$. For $i, j=1, \ldots, n$, define receiving weight $a_{i j}$ and sending weight $b_{i j}$ by

$$
a_{i j}:=\left\{\begin{array}{ll}
1, & v_{j} \in \mathcal{N}_{i}^{\mathrm{I}} \\
0, & v_{j} \notin \mathcal{N}_{i}^{\mathrm{I}}
\end{array} \quad, \quad b_{i j}:= \begin{cases}1, & v_{j} \in \mathcal{N}_{i}^{\mathrm{O}} \\
0, & v_{j} \notin \mathcal{N}_{i}^{\mathrm{O}}\end{cases}\right.
$$

respectively. It is evident that $a_{i j}=b_{j i}$ for $i, j=$ $1, \ldots, n$.

The in-Laplacian matrix $L_{\mathrm{I}}:=\left[l_{i j}^{\mathrm{I}}\right] \in \mathbb{R}^{n \times n}$ and the out-Laplacian matrix $L_{\mathrm{O}}:=\left[l_{i j}^{\mathrm{O}}\right] \in \mathbb{R}^{n \times n}$ are defined according to

$l_{i j}^{\mathrm{I}}:=\left\{\begin{array}{ll}\sum_{k=1}^{n} a_{i k}, & j=i \\ -a_{i j}, & j \neq i\end{array}, l_{i j}^{\mathrm{O}}:= \begin{cases}\sum_{k=1}^{n} b_{i k}, & j=i \\ -b_{i j}, & j \neq i\end{cases}\right.$

respectively. We say that there exists a directed path from $v_{j} \in \mathcal{V}$ to $v_{i} \in \mathcal{V}$ if there are directed edges, i.e.

$\left\{\left(v_{j}, v_{m_{1}}\right),\left(v_{m_{1}}, v_{m_{2}}\right), \ldots,\left(v_{m_{q-1}}, v_{m_{q}}\right),\left(v_{m_{q}}, v_{i}\right)\right\} \subset \mathcal{E}$

for all $1 \leq m_{1}, \ldots, m_{q} \leq n$.

Below are two graphical conditions we shall use later.

Definition 1. The digraph $\mathcal{G}$ is strongly connected if there exists a directed path from any $v_{i}$ to any $v_{j}$ $(i, j=1, \ldots, n, i \neq j)$.

Definition 2. The digraph $\mathcal{G}$ is said to be balanced if $l_{i i}^{\mathrm{I}}=l_{i i}^{\mathrm{O}}$ for every $i=1, \cdots, n$, i.e. the number of in-neighbors of each agent and the number of its out-neighbors are the same.

Note that if $\mathcal{G}$ is the balanced digraph, $L_{\mathrm{I}}=L_{\mathrm{O}}^{\top}$, $L_{\mathrm{I}} \mathbf{1}_{n}=\mathbf{0}_{n}$ and $\mathbf{1}_{n}^{\top} L_{\mathrm{I}}=\mathbf{0}_{n}^{\top}$.

\subsection{Average consensus by surplus- based algorithm}

Let $x_{i}(t) \in \mathbb{R}(i=1, \ldots, n)$ be the state variable of the $i$ th agent, and $x_{i}(0)=x_{i 0}$ the initial value. The average consensus problem requires the state of each agent to converge to the average of the initial values, i.e. $x_{i}(t) \rightarrow(1 / n) \sum_{i=1}^{n} x_{i 0}$ as $t \rightarrow \infty$ for all $i$. 
In the case where a digraph is balanced, the standard algorithm (cf. Olfati-Saber \& Murray, 2004))

$$
\dot{x}_{i}(t)=\sum_{j=1}^{n} a_{i j}\left(x_{j}(t)-x_{i}(t)\right)
$$

is available for the average consensus problem.

To achieve average consensus on arbitrary strongly connected digraphs (not necessarily balanced), Cai and Ishii (2012) proposed a discrete-time surplusbased algorithm. The continuous-time counterpart is as follows:

$$
\begin{aligned}
\dot{x}_{i}(t)= & \sum_{j=1}^{n} a_{i j}\left(x_{j}(t)-x_{i}(t)\right)+\epsilon s_{i}(t) \\
\dot{s}_{i}(t)= & -\sum_{j=1}^{n} a_{i j}\left(x_{j}(t)-x_{i}(t)\right)-\epsilon s_{i}(t) \\
& +\sum_{j=1}^{n}\left(b_{j i} s_{j}(t)-b_{i j} s_{i}(t)\right) \\
x_{i}(0)= & x_{i 0} \in \mathbb{R}, s_{i}(0)=0
\end{aligned}
$$

where $s_{i}(t) \in \mathbb{R}$ is called the surplus variable of the $i$ th agent and $\epsilon>0$ is a parameter which specifies the amount of surplus used in the $x_{i}$ update, and is the parameter of interest as discussed in the introduction. To write (1) in the matrix form, define $x(t):=\left[x_{1}(t) \cdots x_{n}(t)\right]^{\top}, x_{0}:=\left[x_{10} \cdots x_{n 0}\right]^{\top}$, $s(t):=\left[s_{1}(t) \cdots s_{n}(t)\right]^{\top}$, and hence

$$
\begin{gathered}
{\left[\begin{array}{c}
\dot{x}(t) \\
\dot{s}(t)
\end{array}\right]=M\left[\begin{array}{l}
x(t) \\
s(t)
\end{array}\right],\left[\begin{array}{c}
x(0) \\
s(0)
\end{array}\right]=\left[\begin{array}{l}
x_{0} \\
\mathbf{0}_{n}
\end{array}\right]} \\
\text { where } M=\left[\begin{array}{cc}
-L_{\mathrm{I}} & \epsilon I_{n} \\
L_{\mathrm{I}} & -L_{\mathrm{O}}^{\top}-\epsilon I_{n}
\end{array}\right] \in \mathbb{R}^{2 n \times 2 n} .
\end{gathered}
$$

Depending on the digraph, the state $x_{i}(t)$ may diverge when the parameter $\epsilon$ is too large. This is caused by instability of $M$ assocated with larger $\epsilon$. So we need to know whether there is an upper bound on $\epsilon$, and how large its concrete value is if there is the upper bound.

Observe that the column sums of matrix $M$ are zero; thus $\mathbf{1}_{n}^{\top}(\dot{x}(t)+\dot{s}(t))=0$, i.e. $\mathbf{1}_{n}^{\top}(x(t)+s(t))$ is a constant. A convergence result of the surplus-based algorithm (2) is the following.

Lemma 1. Consider the algorithm (2) and suppose that the digraph $\mathcal{G}$ is strongly connected. If the parameter $\epsilon>0$ is sufficiently small, then the agents achieve average consensus.

The proof of Lemma 1 can be found in (Cai, 2011, Corollary 3.2 , p. 33). For completeness we present the proof below.
Proof of Lemma 1. Let

$$
M_{0}=\left[\begin{array}{cc}
-L_{\mathrm{I}} & O_{n} \\
L_{\mathrm{I}} & -L_{\mathrm{O}}^{\top}
\end{array}\right], \quad M_{e}=\left[\begin{array}{cc}
O_{n} & I_{n} \\
O_{n} & -I_{n}
\end{array}\right] .
$$

Then $M=M_{0}+\epsilon M_{e}$; and for strongly connected digraphs the eigenvalues of $M_{0}$ satisfy $0=\lambda_{1}\left(M_{0}\right)=$ $\lambda_{2}\left(M_{0}\right)>\operatorname{Re}\left\{\lambda_{3}\left(M_{0}\right)\right\} \geq \cdots \geq \operatorname{Re}\left\{\lambda_{2 n}\left(M_{0}\right)\right\}$.

Now we qualify the changes of the semi-simple eigenvalue $\lambda_{1}=\lambda_{2}=0$ of $M_{0}$ under a small perturbation $\epsilon M_{e}$. We do this by computing the derivatives $d \lambda_{1}(\epsilon) / d \epsilon$ and $d \lambda_{2}(\epsilon) / d \epsilon$ using (Cai \& Ishii, 2012, Lemma 7$)$; here $\lambda_{1}(\epsilon)$ and $\lambda_{2}(\epsilon)$ are the eigenvalues of $M$ corresponding respectively to $\lambda_{1}$ and $\lambda_{2}$. To that end, choose the right eigenvectors $y_{1}, y_{2}$ and left eigenvectors $z_{1}, z_{2}$ of the semi-simple eigenvalue 0 as follows:

$$
\begin{aligned}
& Y:=\left[\begin{array}{ll}
y_{1} & y_{2}
\end{array}\right]=\left[\begin{array}{cc}
\mathbf{0}_{n} & \mathbf{1}_{n} \\
v_{2} & -n v_{2}
\end{array}\right], \\
& Z:=\left[\begin{array}{l}
z_{1}^{\top} \\
z_{2}^{\top}
\end{array}\right]=\left[\begin{array}{ll}
\mathbf{1}_{n}^{\top} & \mathbf{1}_{n}^{\top} \\
v_{1}^{\top} & \mathbf{0}_{n}^{\top}
\end{array}\right] .
\end{aligned}
$$

Here $v_{1}$ satisfies $v_{1}^{\top} L_{\mathrm{I}}=\mathbf{0}_{n}^{\top}$ and $v_{1}^{\top} \mathbf{1}_{n}=1$; and $v_{2}$ satisfies $L_{\mathrm{O}}^{\top} v_{2}=\mathbf{0}_{n}$ and $\mathbf{1}_{n}^{\top} v_{2}=1$. The fact that positive eigenvectors $v_{1}$ and $v_{2}$ exist follows from the Perron-Frobenius Theorem (Horn \& Johnson, 1990, Chapter 8). With this choice, one can readily verify that $Z Y=I_{2}$. Since $d M /\left.d \epsilon\right|_{\epsilon=0}=M_{e}$, the derivatives $d \lambda_{1}(\epsilon) /\left.d \epsilon\right|_{\epsilon=0}$ and $d \lambda_{2}(\epsilon) /\left.d \epsilon\right|_{\epsilon=0}$ are the eigenvalues of

$$
\left[\begin{array}{cc}
z_{1}^{\top} M_{e} y_{1} & z_{1}^{\top} M_{e} y_{2} \\
z_{2}^{\top} M_{e} y_{1} & z_{2}^{\top} M_{e} y_{2}
\end{array}\right]=\left[\begin{array}{cc}
0 & 0 \\
v_{1}^{\top} v_{2} & -n v_{1}^{\top} v_{2}
\end{array}\right]
$$

In particular, $d \lambda_{1}(\epsilon) /\left.d \epsilon\right|_{\epsilon=0}=0$ and $d \lambda_{2}(\epsilon) /\left.d \epsilon\right|_{\epsilon=0}=$ $-n v_{1}^{\top} v_{2}$. This implies that when $\epsilon$ is small, $\lambda_{1}(\epsilon)$ stays equal to zero while $\lambda_{2}(\epsilon)$ moves to the left along the real axis. Then by continuity, there must exist a positive $\delta_{1}$ such that $\lambda_{1}\left(\delta_{1}\right)=0$ and $\lambda_{2}\left(\delta_{1}\right)<0$. On the other hand, since eigenvalues are continuous functions of its matrix entries, there must also exist a positive $\delta_{2}$ such that $\operatorname{Re}\left\{\lambda_{i}\left(\delta_{2}\right)\right\}<0$ continues to hold for all $i \in\{3, \ldots, 2 n\}$. Thus for any sufficiently small $\epsilon \in\left(0, \min \left\{\delta_{1}, \delta_{2}\right\}\right)$, the matrix $M$ has a simple eigenvalue 0 and all other eigenvalues have negative real part.

Moreover, corresponding to the simple eigenvalue 0 , a right eigenvector is $y_{1}=\left[\begin{array}{ll}\mathbf{1}_{n}^{\top} & \mathbf{0}_{n}^{\top}\end{array}\right]^{\top}$ and a left eigenvector $z_{1}=(1 / n)\left[\mathbf{1}_{n}^{\top} \mathbf{1}_{n}^{\top}\right]^{\top}$. These eigenvectors satisfy $z_{1}^{\top} y_{1}=1$. Therefore

$$
\begin{aligned}
{\left[\begin{array}{l}
x(t) \\
s(t)
\end{array}\right] } & =\mathrm{e}^{t M}\left[\begin{array}{c}
x(0) \\
s(0)
\end{array}\right] \\
& \rightarrow y_{1} z_{1}^{\top}\left[\begin{array}{l}
x(0) \\
s(0)
\end{array}\right]=\left[\begin{array}{l}
\frac{1}{n} \mathbf{1}_{n} \mathbf{1}_{n}^{\top} x(0) \\
\mathbf{0}_{n}
\end{array}\right],
\end{aligned}
$$


as $t \rightarrow \infty$. That is, average consensus is achieved.

In the proof above, observe that by algorithm (2) the multi-agent system achieves average consensus if the matrix $M$ has a simple eigenvalue 0 and all the other eigenvalues have negative real parts. That is,

$$
\begin{aligned}
0 & =\lambda_{1}(M)>\operatorname{Re}\left\{\lambda_{2}(M)\right\} \geq \cdots \geq \operatorname{Re}\left\{\lambda_{2 n}(M)\right\} \\
& \Rightarrow \lim _{t \rightarrow \infty}\left[\begin{array}{l}
x(t) \\
s(t)
\end{array}\right]=\frac{\mathbf{1}_{\mathbf{n}}^{\top} x_{0}}{n}\left[\begin{array}{l}
\mathbf{1}_{n} \\
\mathbf{0}_{n}
\end{array}\right] .
\end{aligned}
$$

By Lemma 1, we know that there always exists a positive $\epsilon$ that ensures convergence to average consensus. A similar result exists for the discrete-time surplus algorithm, and is explored in (Cai \& Ishii, 2012). In particular, provided several results on upper bounds for $\epsilon$ to ensure average consensus, but even for special topologies such as balanced, cyclic, and undirected, the provided bounds are fairly conservative. On the other hand, there is no known bound result for $\epsilon$ for the continuous-time algorithm (2). We fill this gap in this paper, by deriving tight upper bounds on $\epsilon$ that guarantee average consensus over balanced digraphs using algorithm (2).

\section{MAIN RESULT}

Our main result states a tight upper bound on $\epsilon$ for ensuring convergence of algorithm (2) in the case of strongly connected and balanced digraphs.

Theorem 1. Consider the algorithm (2) with the positive parameter $\epsilon$, and suppose that $\mathcal{G}$ is a strongly connected and balanced digraph. Let $\mu_{i}=p_{i}+\mathrm{j} q_{i}$ $(i=1, \ldots, n)$ be the eigenvalues of $L_{\mathrm{I}}$. Then

(i) when there exists at least one eigenvalue of $L_{\mathrm{I}}$ such that $p_{i}<\left|q_{i}\right|$, the agents achieve average consensus if and only if the parameter $\epsilon$ in $M$ satisfies the following inequality

$$
0<\epsilon<\min _{i \in S} \frac{2 p_{i}^{2}}{\left|q_{i}\right|-p_{i}}
$$

where $S:=\left\{k \in[1, n]\left|p_{k}<\right| q_{k} \mid\right\}$;

(ii) when $p_{i} \geq\left|q_{i}\right|$ for all $i=1, \ldots, n$, i.e. $S$ is empty, the agents achieve average consensus if and only if $\epsilon>0$.

Theorem 1 states a tight upper bound on $\epsilon$ for algorithm (2) to achieve average consensus on strongly connected and balanced digraphs. This bound is in

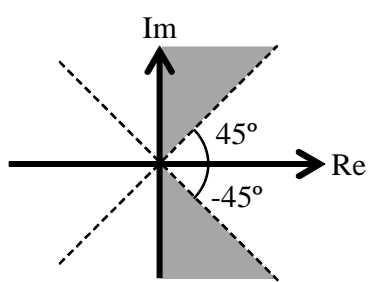

Figure 1: Illustration of condition in Theorem 1

fact needed only when $p_{i}<\left|q_{i}\right|$ holds for some eigenvalue of the in-Laplacian matrix $L_{\mathrm{I}}$, i.e. that eigenvalue lies in the shaded area in Fig. 1.

Compared to the counterpart result in (Cai \& Ishii, 2012) for the discrete-time algorithm on balanced digraphs, the bound in Theorem 1 is tight. This is because, as will be seen below, this bound is proved by directly analyzing the eigenvalues of the matrix $M$, whereas in (Cai \& Ishii, 2012), certain approximation methods were used. Moreover, case (ii) of Theorem 1 where any positive $\epsilon$ works is unique for the continuous-time algorithm; this is not the case for the discrete-time algorithm.

The reason why we can directly analyze the eigenvalues of $M$ is due to the assumption that $\mathcal{G}$ is a balanced digraph and thus $L_{\mathrm{I}}=L_{\mathrm{O}}^{\top}$. We note in passing that the approach of directly analyzing eigenvalues to derive bounds for parameters used in algorithms has been reported in the literature, in particular for consensus problems of agents with second-order dynamics (Ren, 2008; Yu, Chen, \& Cao, 2010).

To prove Theorem 1, we need the following two lemmas. The first lemma provides an expression of eigenvalues of $M$ in terms of those of $L_{\mathrm{I}}$.

Lemma 2. Suppose that $\mathcal{G}$ is a strongly connected and balanced digraph. Let $\mu_{i}:=\lambda_{i}\left(L_{\mathrm{I}}\right) \quad(i=$ $1, \ldots, n)$ be the eigenvalues of $L_{\mathrm{I}}$. Then the eigenvalues of $M$ in algorithm (2) are given as

$$
\lambda_{i}^{ \pm}(M)=\frac{-\left(2 \mu_{i}+\epsilon\right) \pm \sqrt{\epsilon^{2}+4 \mu_{i} \epsilon}}{2}
$$

for $i=1, \ldots, n$.

Proof. Let $\lambda$ and $u=\left[\begin{array}{ll}u_{1}^{\top} & u_{2}^{\top}\end{array}\right]^{\top}$ be an eigenvalue and its associated (right) eigenvector of $M$, respectively. Since $\mathcal{G}$ is a balanced digraph, $L_{\mathrm{I}}=L_{\mathrm{O}}^{\top}$ holds and we 
derive the following:

$$
\begin{aligned}
& M u=\lambda u \\
& \Leftrightarrow\left[\begin{array}{cc}
-L_{\mathrm{I}} & \epsilon I_{n} \\
L_{\mathrm{I}} & -L_{\mathrm{I}}-\epsilon I_{n}
\end{array}\right]\left[\begin{array}{l}
u_{1} \\
u_{2}
\end{array}\right]=\lambda\left[\begin{array}{l}
u_{1} \\
u_{2}
\end{array}\right] \\
& \Leftrightarrow\left\{\begin{array}{l}
-L_{\mathrm{I}} u_{1}+\epsilon u_{2}=\lambda u_{1} \\
L_{\mathrm{I}} u_{1}-L_{\mathrm{I}} u_{2}-\epsilon u_{2}=\lambda u_{2}
\end{array}\right. \\
& \Leftrightarrow\left\{\begin{array}{l}
u_{2}=\frac{1}{\epsilon}\left(\lambda I_{n}+L_{\mathrm{I}}\right) u_{1} \\
L_{\mathrm{I}} u_{1}=\left[(\lambda+\epsilon) I_{n}+L_{\mathrm{I}}\right] u_{2} .
\end{array}\right.
\end{aligned}
$$

From the final two equalities above, we thus obtain

$$
-\left(L_{\mathrm{I}}^{2}+2 \lambda L_{\mathrm{I}}\right) u_{1}=\left(\lambda^{2}+\lambda \epsilon\right) u_{1} .
$$

This means that $\lambda^{2}+\lambda \epsilon$ is an eigenvalue of $-\left(L_{\mathrm{I}}^{2}+\right.$ $\left.2 \lambda L_{\mathrm{I}}\right)$ with an associated eigenvector $u_{1}$. On the other hand, it follows from spectral mapping theorem that $-\left(L_{\mathrm{I}}^{2}+2 \lambda L_{\mathrm{I}}\right)$ has $n$ eigenvalues

$$
-\left(\mu_{1}^{2}+2 \lambda \mu_{1}\right), \ldots,-\left(\mu_{n}^{2}+2 \lambda \mu_{n}\right)
$$

where $\mu_{1}, \ldots, \mu_{n}$ are the eigenvalues of $L_{\mathrm{I}}$. Hence $\lambda^{2}+\lambda \epsilon$ must be equal to one of the eigenvalues in $(6)$, i.e.

$$
\lambda^{2}+\lambda \epsilon=-\left(\mu_{i}^{2}+2 \lambda \mu_{i}\right),
$$

for some $i \in\{1, \ldots, n\}$. This quadratic equation has two roots. By solving the equation for $\lambda$ and denoting the two roots as $\lambda_{i}^{ \pm}$, we obtain (5).

In the sequel we shall use the notation $\lambda_{i}^{+}(M)$ and $\lambda_{i}^{-}(M)$, where $\lambda_{i}^{+}(M)$ is the ' + ' side of \pm , and $\lambda_{i}^{-}(M)$ is the ' - ' side. The second lemma states a useful expression for the real part of the square root of a complex number.

Lemma 3. Let $v_{1}, v_{2}$ be any real numbers. Then

$$
\operatorname{Re}\left\{ \pm \sqrt{v_{1}+\mathrm{j} v_{2}}\right\}= \pm \sqrt{\frac{v_{1}+\sqrt{v_{1}^{2}+v_{2}^{2}}}{2}} .
$$

Proof. Let $w_{1}, w_{2}$ be real numbers satisfying $\pm \sqrt{v_{1}+\mathrm{j} v_{2}}= \pm\left(w_{1}+\mathrm{j} w_{2}\right)$. Then the square of both sides of the above equation is $v_{1}+\mathrm{j} v_{2}=\left(w_{1}^{2}-w_{2}^{2}\right)+$ $\mathrm{j} 2 w_{1} w_{2}$ and we derive the following:

$$
\begin{aligned}
\left\{\begin{array}{l}
v_{1}=w_{1}^{2}-w_{2}^{2} \\
v_{2}=2 w_{1} w_{2}
\end{array}\right. & \Leftrightarrow\left\{\begin{array}{l}
w_{1}^{2}=v_{1}+w_{2}^{2} \\
w_{2}=\frac{v_{2}}{2 w_{1}}
\end{array}\right. \\
& \Leftrightarrow w_{1}^{2}=v_{1}+\frac{v_{2}^{2}}{4 w_{1}^{2}} \\
& \Leftrightarrow 4\left(w_{1}^{2}\right)^{2}-4 v_{1} w_{1}^{2}-v_{2}^{2}=0 \\
& \Leftrightarrow w_{1}^{2}=\frac{v_{1} \pm \sqrt{v_{1}^{2}+v_{2}^{2}}}{2} .
\end{aligned}
$$

Since $w_{1}^{2}$ in the last equation above cannot be negative, $w_{1}^{2}=\left(v_{1}+\sqrt{v_{1}^{2}+v_{2}^{2}}\right) / 2$. From $\operatorname{Re}\left\{ \pm \sqrt{v_{1}+\mathrm{j} v_{2}}\right\}=w_{1}$ we obtain (7).

Now we are ready to prove Theorem 1 .

Proof of Theorem 1. Since $\mu_{i}=p_{i}+\mathrm{j} q_{i}$ are the eigenvalues of $L_{\mathrm{I}}$, which is associated with a strongly connected digraph, we can order $\mu_{i}$ such that $p_{i}$ and $q_{i}$ satisfy $0=p_{1}<p_{2} \leq \cdots \leq p_{n}$ and $q_{1}=0$, respectively (Mesbahi \& Egerstedt, 2010). By substituting $\mu_{i}=p_{i}+\mathrm{j} q_{i}$ in equation (5) of Lemma 2, we derive

$$
\lambda_{i}^{ \pm}(M)=\frac{-\left[\left(2 p_{i}+\epsilon\right)+\mathrm{j} 2 q_{i}\right] \pm \sqrt{\epsilon r_{i}+\mathrm{j} 4 q_{i} \epsilon}}{2}
$$

where $r_{i}=\epsilon+4 p_{i}$. When $i=1$,

$$
\lambda_{1}^{ \pm}(M)=\frac{-\epsilon \pm \epsilon}{2} \Rightarrow\left\{\begin{array}{l}
\lambda_{1}^{+}(M)=0 \\
\lambda_{1}^{-}(M)=-\epsilon<0 .
\end{array}\right.
$$

Thus $M$ has at least one 0 eigenvalue.

Using Lemma 3, we obtain

$\operatorname{Re}\left\{\lambda_{i}^{ \pm}(M)\right\}=\frac{-\left(2 p_{i}+\epsilon\right) \pm \sqrt{\frac{\epsilon r_{i}+\epsilon \sqrt{r_{i}^{2}+16 q_{i}^{2}}}{2}}}{2}$.

Since $M$ has at least one 0 eigenvalue, it is left to find an upper bound of $\epsilon>0$ to ensure $\operatorname{Re}\left\{\lambda_{i}^{ \pm}(M)\right\}<0$ for $i=2, \ldots, n$ (owing to (3)). From (8), we see that $\operatorname{Re}\left\{\lambda_{i}^{-}(M)\right\}<0$ holds for $i=2, \ldots, n$ regardless the value of $\epsilon$. Thus it suffices to derive an upper bound of $\epsilon$ which guarantees $\operatorname{Re}\left\{\lambda_{i}^{+}(M)\right\}<0$ and towards this end we derive the following:

$$
\begin{aligned}
-\left(2 p_{i}\right. & +\epsilon)+\sqrt{\frac{\epsilon r_{i}+\epsilon \sqrt{r_{i}^{2}+16 q_{i}^{2}}}{2}}<0 \\
& \Leftrightarrow \epsilon r_{i}+\epsilon \sqrt{r_{i}^{2}+16 q_{i}^{2}}<2\left(2 p_{i}+\epsilon\right)^{2} \\
& \Leftrightarrow \epsilon^{2} r_{i}^{2}+16 q_{i}^{2} \epsilon^{2}<\left(2\left(2 p_{i}+\epsilon\right)^{2}-\epsilon r_{i}\right)^{2} \\
& \Leftrightarrow 16 p_{i}^{2} \epsilon^{2}+16 q_{i}^{2} \epsilon^{2}<32 p_{i}^{2} \epsilon^{2}+64 p_{i}^{3} \epsilon+64 p_{i}^{4} \\
& \Leftrightarrow\left(p_{i}^{2}-q_{i}^{2}\right) \epsilon^{2}+4 p_{i}^{3} \epsilon+4 p_{i}^{4}>0
\end{aligned}
$$

for all $i=2, \ldots, n$. Then we have the following cases.

a) When $p_{i}=\left|q_{i}\right|$, it is clear that inequality (9) holds if and only if $\epsilon>0$.

b) When $p_{i}>\left|q_{i}\right|$, the solution of (9) is

$$
\epsilon<\frac{-2 p_{i}^{2}}{p_{i}-\left|q_{i}\right|}, \quad \epsilon>\frac{-2 p_{i}^{2}}{p_{i}+\left|q_{i}\right|} .
$$

This means that inequality (9) holds if and only if $\epsilon>0$. 
c) When $p_{i}<\left|q_{i}\right|$, the solution of (9) is

$$
-\frac{2 p_{i}^{2}}{\left|q_{i}\right|+p_{i}}<\epsilon<\frac{2 p_{i}^{2}}{\left|q_{i}\right|-p_{i}} .
$$

Since $\epsilon>0$, inequality (9) holds if and only if

$$
0<\epsilon<\min _{i} \frac{2 p_{i}^{2}}{\left|q_{i}\right|-p_{i}} .
$$

By a) and b), condition ii) of Theorem 1 is proved and by c), condition i) of Theorem 1 is proved.

\section{SPECIAL TOPOLOGIES}

We investigate the following special graphs using Theorem 1, to obtain specialized results. In doing so, we obtain insight into possible requirements of $\epsilon$ for general strongly connected digraphs.

\subsection{Connected undirected graph}

A connected undirected graph has bidirectional edges and there is a (bidirectional) path between any pair of nodes. The in-Laplacian matrix $L_{\mathrm{I}}$ associated with this graph is a symmetric matrix, so all of its eigenvalues are real.

Proposition 1. Consider algorithm (2) with the positive parameter $\epsilon$, and suppose that $\mathcal{G}$ is a connected undirected graph. Then the agents achieve average consensus if and only if $\epsilon>0$.

Proposition 1 asserts that algorithm (2) achieves average consensus on undirected graphs regardless of the value of parameter $\epsilon(>0)$. This is in sharp contrast with the counterpart bound result given in (Cai \& Ishii, 2012) for the discrete-time algorithm which cannot be arbitrary and is dependent on the number $n$ of agents.

The proof of Proposition 1 is as follows.

Proof of Proposition 1. Since all eigenvalues of $L_{\mathrm{I}}$ are real, all the imagnary part are zero and thus $S$ defined in (4) is empty. This condition matches case (ii) of Theorem 1, hence the agents achieve average consensus if and only if $\epsilon>0$.

\subsection{Cyclic digraph}

An (unweighted) cyclic digraph is one of the simplest strongly connected digraphs. This digraph $\mathcal{G}$ has a node set $\mathcal{V}=\left\{v_{1}, \ldots, v_{n}\right\}$ and an edge set $\mathcal{E}=\{(1,2),(2,3), \ldots,(n-1, n),(n, 1)\}$. Moreover

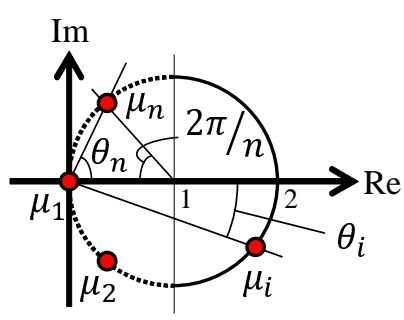

Figure 2: Illustration of eigenvalue distribution for in-Laplacian matrix of cyclic digraph

$L_{\mathrm{I}}$ is a circulant matrix, so the eigenvalues $\mu_{i}$ of $L_{\mathrm{I}}$ satisfy

$$
\mu_{i}=1-\cos \frac{2(i-1)}{n} \pi-\mathrm{j} \sin \frac{2(i-1)}{n} \pi
$$

for $i=1, \cdots, n$ (cf. Agaev \& Chebotarev, 2010, Theorem 1)). Fig. 2 shows the spectrum of $L_{\mathrm{I}}$ of $n$ nodes uniformly distributed on a circle centered at 1 . Number the eigenvalues $\mu_{1}, \ldots, \mu_{n}$ counterclockwise, beginning with $\mu_{1}=0$ and define $\theta_{n} \in[0,2 \pi), n \geq 2$ to be the angle between the line going through $\mu_{1}$ and $\mu_{n}$ and the real axis. From Fig. 2, $\theta_{n}$ satisfies

$$
\begin{gathered}
2 \theta_{n}+\frac{2 \pi}{n}=\pi \\
\Rightarrow \theta_{n}=\left(\frac{1}{2}-\frac{1}{n}\right) \pi .
\end{gathered}
$$

If some eigenvalue $\mu_{i}=p_{i}+\mathrm{j} q_{i}$ is on the dotted arc as shown in Fig. 2, then $\mu_{i}$ is such that $p_{i}<\left|q_{i}\right|$ (i.e. case (i) of Theorem 1).

Proposition 2. Consider algorithm (2) with the positive parameter $\epsilon$, and suppose that $\mathcal{G}$ is a cyclic digraph. Then

(i) when $2 \leq n \leq 4$, the agents achieve average consensus if and only if $\epsilon>0$;

(ii) when $n \geq 5$, the agents achieve average consensus if and only if the parameter $\epsilon$ in $M$ satisfies the following inequality

$$
0<\epsilon<\frac{4}{\tan ^{3} \theta_{n}-\tan ^{2} \theta_{n}+\tan \theta_{n}-1}
$$

where $\theta_{n}$ is defined in (10).

Proposition 2 states a tight upper bound on $\epsilon$ for algorithm (2) to achieve average consensus over cyclic digraphs with $n \geq 5$ agents (when $2 \leq n \leq 4$ any positive $\epsilon$ works). By contrast, the counterpart bound result in (Cai \& Ishii, 2012) for the discrete-time algorithm is not tight, because it is derived based on a 
matrix perturbation result with approximations. In addition, there is no case (including $n=2,3,4$ ) in (Cai \& Ishii, 2012) where $\epsilon$ can be unbounded.

Note that the specialized bound in (11) is determined by just one parameter, $\theta_{n}$ in (10), which makes this bound easier to calculate than the general bound in (4). More significantly, we conjecture in Section 5.3, using extensive simulations as ev dence, that the bound in (11) for cyclic digraphs likely to be the 'worst-case' bound, in the sense the if this bound is satisfied by parameter $\epsilon$, algorithı (2) might achieve average consensus over arbitrar strongly connected digraphs including non-balance ones.

The proof of Proposition 2 is as follows.

Proof of Proposition 2. Let $\mu_{i}=p_{i}+\mathrm{j} q_{i}$ for $i=$ $1, \ldots, n$ be eigenvalues of $L_{\mathrm{I}}$. When $2 \leq n \leq 4$, since $p_{i} \geq\left|q_{i}\right|$ for all $i=1, \ldots, n$, therefore by Theorem 1 the agents achieve average consensus if and only if $\epsilon>0$. When $n \geq 5$, the set $S$ in (4) is not empty. We define a quantity $\theta_{i}$ for the angle (in polar coordinates) of the complex number $\mu_{i}$ (see Fig. 2), i.e.

$$
\theta_{i}:=\arg \mu_{i}=\tan ^{-1}\left(\frac{\operatorname{Im}\left(\mu_{i}\right)}{\operatorname{Re}\left(\mu_{i}\right)}\right), i \in S .
$$

Then $\pi / 4<\left|\theta_{i}\right|<\pi / 2$ holds for any $i \in S$. Because all the eigenvalues are on a circular arc, the following are derived using (12):

$$
p_{i}=2 \cos ^{2} \theta_{i}, q_{i}=2 \sin \theta_{i} \cos \theta_{i}, i \in S .
$$

By substituting (13) into equation (4) and simplifying the equation, we obtain the upper bound $\bar{\epsilon}$ on parameter $\epsilon$ :

$$
\begin{aligned}
\bar{\epsilon} & =\min _{i \in S} \frac{8 \cos ^{4} \theta_{i}}{2\left|\sin \theta_{i} \cos \theta_{i}\right|-2 \cos ^{2} \theta_{i}} \\
& =\min _{i \in S} \frac{4 \cos ^{2} \theta_{i}}{\left|\tan \theta_{i}\right|-1} \\
& =\min _{i \in S} \frac{4}{\left(\left|\tan \theta_{i}\right|-1\right)\left(\tan ^{2} \theta_{i}+1\right)}
\end{aligned}
$$

Here $\left|\tan \theta_{i}\right|>1$ for all $i \in S$. To minimize (14), the denominator needs to be maximized. Because $i \in S$, it is observed that the angle $\left|\theta_{i}\right|$ which is closest to $\pi / 2$ maximizes $\left|\tan \theta_{i}\right|$ and thus the denominator. Based on how the eigenvalues are numbered (counterclockwise from $\left.\mu_{1}=0\right)$, such an angle is $\theta_{n}$ and $\theta_{n}$ minimizes (14). Therefore we derive (11).

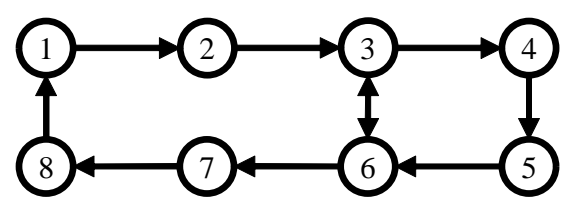

Figure 3: A balanced digraph

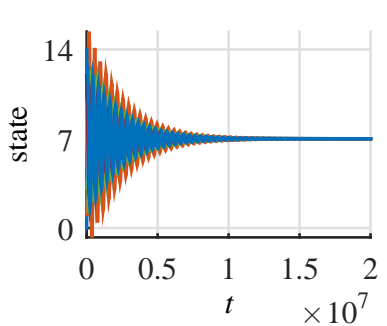

(a)

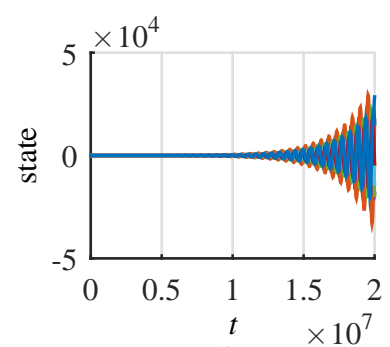

(b)
Figure 4: Convergence results on the balanced digraph (Fig. 3): (a) $\epsilon=\bar{\epsilon}-0.0001$ and (b) $\epsilon=$ $\bar{\epsilon}+0.0001$

\section{NUMERICAL EXAMPLES}

We illustrate by simulations the derived bound results, as well as the extension to unbalanced strongly connected digraphs.

\subsection{Balanced digraph}

As an example of a balanced digraph, we use the digraph in Fig. 3 consisting of 8 nodes. The eigenvalues of $L_{\mathrm{I}}$ in algorithm (2) associated with this digraph are

$$
\begin{aligned}
0,0.5331 & \pm 0.6588 \mathrm{j}, 1.1574 \pm 0.8487 \mathrm{j}, \\
1.7439 & \pm 0.6429 \mathrm{j}, 3.1311
\end{aligned}
$$

and there exist eigenvalues satisfying $p_{i}<\left|q_{i}\right|$; in particular $S=\{0.5331 \pm 0.6588 \mathrm{j}\}$. Then the upper bound of $\epsilon$ is $\bar{\epsilon}=4.5231$ by Theorem 1 .

We show the convergence result with $\epsilon=\bar{\epsilon}-$ $0.0001=4.5230$ and $\epsilon=\bar{\epsilon}+0.0001=4.5232$ in Fig. 4 (a) and (b), respectively. The initial states of each agents are $x_{i 0}=2(i-1), i=1, \ldots, 8$. When $\epsilon=4.5230$, the states of all agents approach to the average of the initial states which is $(1 / 8) \mathbf{1}_{8}^{\top} x_{0}=7$, but they diverge when $\epsilon=4.5232$. This demonstrates the tightness of the bound we derived for $\epsilon$.

\subsection{Cyclic digraphs}

We deal with cyclic digraphs in which the number of nodes are $n=3, \ldots, 8$. Table 1 shows $\theta_{n}$ in (10) and the upper bound $\bar{\epsilon}$ calculated from Proposition 2 . 
Table 1: Upper bound of $\epsilon$ on cyclic digraphs with $n$ nodes

\begin{tabular}{|c||c|c|c|c|c|}
\hline$n$ & 3 & 4 & 5 & 6 & 7 \\
\hline$\theta_{n}$ & $(1 / 6) \pi$ & $(1 / 4) \pi$ & $(3 / 10) \pi$ & $(1 / 3) \pi$ & $(5 / 14) \pi$ \\
\hline $\bar{\epsilon}$ & $\infty$ & $\infty$ & 3.6717 & 1.3660 & 0.6995 \\
\hline
\end{tabular}

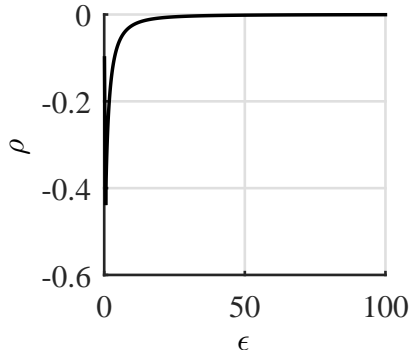

(a)

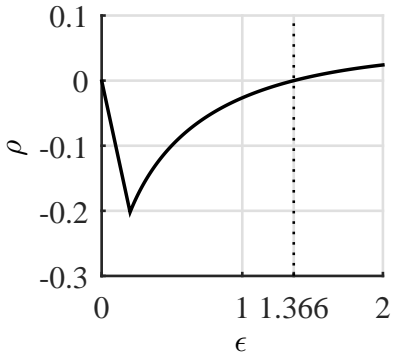

(b)
Figure 5: Maximum real part $\rho$ of the eigenvalues of $M$ except for the 0 eigenvalue on cyclic digraphs: (a) $n=4$ and (b) $n=6$

Observe that $\bar{\epsilon}$ decreases as $n$ increases. Define $\rho$ as the maximum real part of the eigenvalues of $M$, and Fig. 5 (a) and (b) show $\rho$ in algorithm (2) as $\epsilon$ is varied, except for $\rho=0$ with $n=4$ and $n=6$, respectively. If $\rho<0$, average consensus is achieved by algorithm (2). Observe that $\rho<0$ holds even if $\epsilon$ increases to 100 for $n=4$ (case (i) of Proposition 2), while $\rho>0$ from $\epsilon \geq 1.3660$ for $n=6$ (case (ii) of Proposition 2).

\subsection{Unbalanced strongly connected digraphs}

Consider a cyclic digraph of 8 nodes, and we now randomly add some directed edges. There are in total $48(=8 \times 7-8)$ directed edges that are candidates to be added. From these 48 candidates, select $m(\in\{1, \ldots, 48\})$ edges uniformly at random and add them to the cyclic digraph. Then numerically compute the upper bound $\epsilon_{m}$, which is the maximal value of $\epsilon$ such that the maximum real part $\rho$ of eigenvalues of $M$ (expect for the 0 eigenvalue) satisfies $\rho<0$. If $\epsilon_{m}>40$ then set $\epsilon_{m}=40$, because this value is already much larger than $0.4142\left(=: \epsilon_{0}\right)$ computed in the preceding subsection as the upper bound for a cyclic digraph of 8 nodes. Repeat the above 200 times for the same $m$, and calculate $\epsilon_{\max }$ as the maximum of $\epsilon_{m}, \epsilon_{\min }$ as the minimum and $\epsilon_{\text {ave }}$ as the average for each $m$.

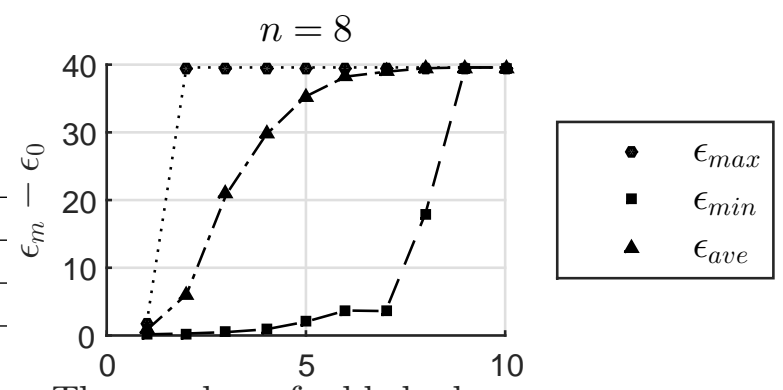

The number of added edges $m$

Figure 6: Upper bound comparison between unbalanced digraphs and cyclic digraphs $(n=8)$

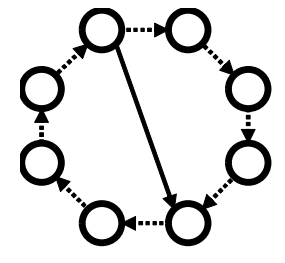

(a)

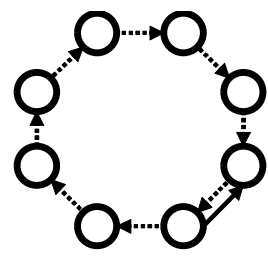

(b)
Figure 7: Unbalanced digraphs with one edge added. (a) generates the maximum $\epsilon_{\max }$ (see Fig. 6 for $m=$ 1); (b) generates the minimum $\epsilon_{\min }$ (see Fig. 6 for $m=1)$

In Fig. 6 we plot the differences $\epsilon_{\max }-\epsilon_{0}, \epsilon_{\min }-\epsilon_{0}$ and $\epsilon_{\text {ave }}-\epsilon_{0}$ for different number $m$ of edges added. For $m>10$ the differences are all larger than 40, and thus omitted from the figure. The fact that $\epsilon_{\min }-\epsilon_{0}>0$ means that average consensus is achieved for all simulated digraphs if $\epsilon_{0}$ is used. In other words, the upper bounds on parameter $\epsilon$ for the randomly generated digraphs (strongly connected and possibly unbalanced) are larger than the bound for cyclic digraphs, and indeed, the more edges are added the larger the bound on $\epsilon$ becomes.

We have performed the same simulation as above for initially cyclic digraphs of 9 and 10 nodes (with directed edges randomly added), and similar results are observed. This suggests that cyclic digraphs might be the 'worst-case' for convergence of the surplus-based algorithm, in the sense that as long as the parameter satisfies the (tight) bound for cyclic digraphs, this parameter can work for other possibly unbalanced digraphs.

Another interesting observation is that the 'longer distance' of two nodes between which a directed edge is added, the larger $\epsilon_{m}$ is. Here distance of two nodes refers to the minimal number of directed edges from one node to the other on the initial cyclic digraph. Fig. 7 shows two unbalanced digraphs obtained from 


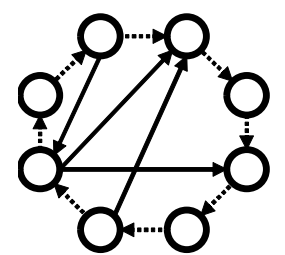

(a)

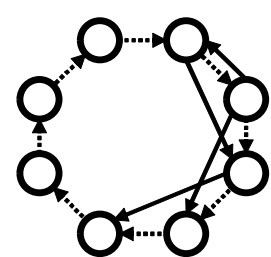

(b)
Figure 8: Unbalanced digraphs with four edges added. (a) generates the maximum $\epsilon_{\max }$ (see Fig. 6 for $m=4$ ); (b) generates the minimum $\epsilon_{\min }$ (see Fig. 6 for $m=4$ )

a cyclic digraph of 8 nodes with one edge added; the dotted arrows are edges of the initial cyclic digraph, while the solid arrow is the added edge. In Fig. 7 (a) the distance of two nodes where the edge is added is 4, and in this case the maximum $\epsilon_{\max }$ (see Fig. 6 for $m=1$ ) is generated. By contrast, in Fig. 7 (b) the distance is 1 and the minimum $\epsilon_{\min }$ (see Fig. 6 for $m=1$ ) is generated.

As another example confirming the same observation, Fig. 8 shows two unbalanced digraphs with four edges added. The maximum $\epsilon_{\max }$ (see Fig. 6 for $m=4$ ) is generated by the digraph in Fig. 8 (a) with the average distance 3 of two nodes where an edge is added, whereas the minimum $\epsilon_{\min }$ (see Fig. 6 for $m=4$ ) is generated by the digraph in Fig. 8 (b) with the average distance 1.75 . The above observations suggest that the edges added between nodes of longer distance can generate a larger upper bound on the parameter $\epsilon$.

\section{CONCLUSIONS}

We have studied the continuous-time surplus-based algorithm, and derived a tight upper bound on the parameter included in this algorithm over strongly connected and balanced digraphs. The result guarantees that the states of all agents in the network converge to the average of their initial states when the parameter is smaller than the upper bound. Furthermore, we have specialized the upper bound result for undirected graphs and cyclic digraphs. In particular, for undirected graphs, any $\epsilon>0$ ensures convergence and for cyclic digraphs, the upper bound on $\epsilon$ depends only on the number of agents.

In future work, we are interested in deriving upper bounds on parameter $\epsilon$ for general strongly connected digraphs (possibly unbalanced); in particular we aim to prove the observation, made using simulations, in Section 5.3. We also aim to characterize the relation between parameter $\epsilon$ and the convergence speed of algorithm (2).

\section{References}

Agaev, R., \& Chebotarev, P. (2010). Which digraphs with ring structure are essentially cyclic? $A d$ vances in Applied Mathematics, 45(2), 232251.

Bénézit, F., Blondel, V., Thiran, P., Tsitsiklis, J., \& Vetterli, M. (2010). Weighted gossip: Distributed averaging using non-doubly stochastic matrices. In 2010 IEEE International Symposium on Information Theory (pp. 1753-1757).

Boyd, S., Ghosh, A., Prabhakar, B., \& Shah, D. (2006). Randomized gossip algorithms. IEEE Transactions on Information Theory, 52(6), 2508-2530.

Cai, K. (2011). Multi-agent consensus and averaging on general network topology. Doctoral Dissertation, Tokyo Insititute of Technology.

Cai, K. (2012). Averaging over general random networks. IEEE Transactions on Automatic Control, 57(12), 3186-3191.

Cai, K., \& Ishii, H. (2012). Average consensus on general strongly connected digraphs. Automatica, 48(11), 2750-2761.

Cai, K., \& Ishii, H. (2014). Average consensus on arbitrary strongly connected digraphs with timevarying topologies. IEEE Transactions on Automatic Control, 59(4), 1066-1071.

Cao, Y., Yu, W., Ren, W., \& Chen, G. (2013). An overview of recent progress in the study of distributed multi-agent coordination. IEEE Transactions on Industrial Informatics, 9(1), 427-438.

Horn, R. A., \& Johnson, C. R. (1990). Matrix Analysis. Cambridge: Cambridge University Press.

Jadbabaie, A., Lin, J., \& Morse, A. S. (2003). Coordination of groups of mobile autonomous agents using nearest neighbor rules. IEEE Transactions on Automatic Control, 48(6), 988-1001.

Mesbahi, M., \& Egerstedt, M. (2010). Graph Theoretic Methods in Multiagent Networks. New Jersey: Princeton University Press.

Olfati-Saber, R., \& Murray, R. M. (2004). Consensus problems in networks of agents with switching topology and time-delays. IEEE Transactions on Automatic Control, 49(9), 1520-1533.

Qin, J., Ma, Q., Shi, Y., \& Wang, L. (2017). Recent advances in consensus of multi-agent systems: 
A brief survey. IEEE Transactions on Industrial Electronics, 64(6), 4972-4983.

Ren, W. (2008). On consensus algorithms for doubleintegrator dynamics. IEEE Transactions on Automatic Control, 53(6), 1503-1509.

Xiao, L., \& Boyd, S. (2004). Fast linear iterations for distributed averaging. Systems \& Control Letters, 53, 65-78.

Xu, Y., Han, T., Cai, K., Lin, Z., Yan, G., \& Fu, M. (2017). A distributed algorithm for resource allocation over dynamic digraphs. IEEE Transactions on Signal Processing, 65(10), 26002612.

Yu, W., Chen, G., \& Cao, M. (2010). Some necessary and sufficient conditions for second-order consensus in multi-agent dynamical systems. $A u-$ tomatica, 46(6), 1089-1095. 